\title{
Circumstances attending roo sudden deaths from coronary artery disease with coroner's necropsies
}

\author{
A. Myers $^{1}$ and H. A. Dewar \\ From the Royal Victoria Infirmary, Newcastle upon Tyne
}

The deaths of 100 men due to coronary artery disease which occurred so suddenly and unexpectedly as to merit a coroner's necropsy have been studied, with special reference to the exact circumstances of their occurrence. The most significant relationship of sudden death was with acute psychological stress. Moderate physical activity, the time of day, the day of the week, and a recent meal, especially if accompanied by alcohol, were also significantly related. Very strenuous exercise, the season of the year, the environmental temperature or recent change of it, and chronic psychological stress were not so related. Neither were the actual smoking of a cigarette nor the composition of the meal immediately preceding death.

Compared with previous series of proved acute myocardial infarction the necropsies in these cases showed that the right coronary artery had been recently occluded by a thrombus more often than the left anterior descending. Stenosis or occlusion of the right coronary artery bore a significant relation to the suddenness of death. Special analysis of the 52 cases in which neither recent thrombus nor infarction were found did not disclose any circumstances attending death which differed from the remainder.

Some comparisons are made with the circumstances attending the onset of symptoms in roo men studied while recovering from an acute myocardial infarct.

This study was designed to provide up to date in- $\$$ infarction, or both. Previous post-mortem studies formation on the circumstances which attend sudden deaths due to coronary heart disease in an urban community in the U.K., and especially to try to determine to what extent these circumstances could be considered to be causally related to the deaths and to the post-mortem findings in the heart. For this purpose 100 consecutive such deaths in Newcastle upon Tyne were taken. They were sufficiently sudden and unexpected as to be referred to the coroner, and because of this referral, necropsies were performed in every instance. It was from his office and with his co-operation that the names and addresses were obtained. All the roo cases fulfilled the WHO definition of sudden death as death occurring within 24 hours of the onset of acute symptoms. The duration of symptoms before death was as follows: none (instantaneous), 34 ; less than I5 minutes, 20; 15 minutes to one hour, 19; more than one hour, 27. In only eight of the cases was there time for help to be obtained.

The necropsy provided evidence on whether there had been coronary thrombosis or myocardial

Received 28 April 1975.

1 Present address: Royal Free Hospital, London. (French and Dock, 1944; Yater et al., 1948; Crawford, Dexter, and Teare, 196I ; Baroldi, 1965; Hansen, 1968; Spain and Bradess, 1970; Wikland, 1971 ; Friedman et al., 1973) have shown that in nearly every case severe coronary atheroma will be found, but that very many fewer will show evidence of recent coronary thrombosis or myocardial infarction, or both. It is by no means certain that when neither has been found neither has been present, for thrombi may disintegrate or lyse after death and infarcts may be too fresh to be recognizable by any staining method. Spain and Bradess (1970), however, from careful studies sometimes made as early as 15 minutes after death, concluded that this was not a likely explanation and noted that in the sudden cases where an infarct but no thrombus had been found the infarct was usually multifocal and subendocardial, whereas when a thrombus was present the infarct was unifocal and transmural. Consistent with this are the observations of Haerem (1971), recorded after this investigation had begun, that many patients who died suddenly and unexpectedly had platelet aggregates occluding their intramyocardial vessels. Presumably in the presence of a circulation slowed by coronary stenoses factors 
which enhance platelet aggregability can operate and cause death either by inducing multifocal infarction or by inducing widespread ischaemia leading to death from ventricular fibrillation or asystole. Adrenaline and noradrenaline had already been shown by Mitchell and Sharp (1964) to be potent agents of such platelet clumping.

In this inquiry we paid particular attention to the patient's normal habits and mode of life as well as to the circumstances in which he died, so as to determine as far as possible whether these circumstances were unusual for him or of more than coincidental significance. In this sense, therefore, each subject provided his own control of long uneventful periods of time. The results of the inquiry have been analysed statistically from this point of view.

Nevertheless, in addition roo cases of proved but non-fatal infarction were also studied by a technique very similar to but not identical with that of the sudden deaths. Some comparison has been made between these cases and the sudden fatalities with the special end in view of trying to determine if there were circumstances in the sudden death cases in whom fatal arrhythmias without thrombosis or infarction occurred in significant numbers and which were absent from the non-fatal infarct series. This will be brought out in the analysis.

\section{Subjects and methods}

Only males of 70 years or less were included in the study. The relatives in each case were visited unannounced by A.M. between five and ro days after the death. With their help and with the help of others, if any, who had witnessed the death or the events leading to it a standard questionnaire was completed. This, in addition to giving details of age, occupation, past medical history, and family history, also recorded any unusual exercise that had been taken before death, any unusual psychological stress that had occurred, what meals had been taken before death and what were the subject's usual dietary habits, what was his smoking habit and what he had been smoking, if at all, just before he died, and what was his customary alcohol consumption and what had been consumed before the fatality. The environmental circumstances of death, especially the temperature and any recent change of temperature, were also recorded. In only one instance did the relatives refuse to co-operate, and that case was excluded from the series. In 88 cases the moment of death was actually witnessed. Seven died in their sleep, either with someone else in the room or so close that any cry or complaint would have been heard. These are classified as instantaneous but unwitnessed. Five others also died unwitnessed, though they had been seen alive not more than one hour previously; two of them were found dead in bed with clothing undisturbed, one died in a lavatory, one in a corridor, and one very close in front of a fire. These are also classified as instantaneous but unwitnessed.

In every case a coroner's necropsy was performed. In Newcastle upon Tyne coroner's necropsies are performed in one of the two main hospitals, the Royal Victoria Infirmary or the Newcastle General Hospital, and with few exceptions by one of two pathologists. Agreement was reached for the pathologist to perform the examination of the heart in a standard manner with 5-mm transverse sectioning of the three main coronary arteries. Details were recorded of the degree of atheroma and obstruction as a percentage occlusion of the vessel, the position of the narrowing was recorded in centimetres from the origin of that vessel, and the nature of obstruction in those vessels was described, especially whether it was by atheroma or by new or old thrombus. The presence and site of any obvious old or new myocardial infarct were also noted.

Macroscopic staining with triphenyl tetrazolium chloride (by the method of Camps (1969) was used. Since infarcted myocardium of five hours' duration or more does not stain with this reagent suspicious areas of recent infarction could be delineated. Specimens of these and of any other areas of old or obvious new infarction were taken and slides made from them and stained with Van Gieson stain and with haematoxylin and eosin. These slides were all reported on by one pathologist.

For the 'controls' IOO men were used who were not over the age of 70 and who had recently been admitted to the Royal Victoria Infirmary with a myocardial infarct confirmed by electrocardiogram or enzyme estimation, or both. These were not quite consecutive admissions because some patients died before they could be questioned and patients where the diagnosis was in any doubt were excluded. The same standard questionnaire was completed with the help of the patients and their relatives. Patients and relatives were seen separately and if different answers were given to any question those of the latter were accepted and recorded. This was done in order to make the comparison with the sudden death group as valid as possible. The end point for the infarct group was taken as the time of onset of severe symptoms which led to the diagnosis of myocardial infarction. For this reason 'silent' infarcts were not included in this control group.

The results were tabulated in a form suitable for computer analysis. This analysis was carried out by the Department of Medical Statistics of the University of Newcastle upon Tyne, using a University of Michigan terminal system (model AW.I20). The significance of the results was worked out on $\chi^{2}$ tables. Where frequencies in each group can be compared with an expected distribution - for example, the day of the week or the hour of the day, etc. - they should have more validity than the comparison between the sudden death and non-fatal myocardial infarct groups. The $P$ values should be assessed accordingly.

\section{Results}

The personal details of the roo cases of sudden death are set out in Table $\mathbf{I}$ with comparable details 
TABLE I Comparison of sudden death and non-fatal myocardial infarct groups (100 cases in each)

\begin{tabular}{|c|c|c|c|}
\hline & Sudden death & $\begin{array}{l}\text { Non-fatal } \\
\text { myocardial } \\
\text { infarction }\end{array}$ & Significance \\
\hline \multicolumn{4}{|l|}{ Age } \\
\hline $\begin{array}{l}\text { mean } \\
\text { range }\end{array}$ & $\begin{array}{l}57.4 \\
35-70\end{array}$ & $\begin{array}{l}54.2 \\
27-70\end{array}$ & NS \\
\hline Weight (ponderal index) & Norma! & $7 \%$ excess & $P<0.01$ \\
\hline \multicolumn{4}{|l|}{ Social class } \\
\hline I & $\mathbf{I}$ & 5 & \multirow{5}{*}{$P<0$ O I } \\
\hline II & 7 & 20 & \\
\hline III & 47 & 53 & \\
\hline IV & 21 & 15 & \\
\hline V & 24 & 7 & \\
\hline \multicolumn{4}{|l|}{ Smoking habits } \\
\hline non-smokers & 15 & 18 & \multirow{4}{*}{$\begin{array}{l}\text { NS } \\
\text { NS } \\
\text { NS } \\
\text { NS }\end{array}$} \\
\hline pipe or cigars only & IO & 12 & \\
\hline up to 15 cigarettes/day & 30 & 29 & \\
\hline more than 15 cigarettes/day & 45 & $4 I$ & \\
\hline \multicolumn{4}{|l|}{ Alcohol habits } \\
\hline non-drinkers & 23 & 18 & \multirow{6}{*}{$\mathbf{N S}$} \\
\hline seldom & I3 & 15 & \\
\hline daily & 17 & 19 & \\
\hline weekends only & 43 & 45 & \\
\hline weekly (not weekends) & 8 & & \\
\hline $\begin{array}{l}\text { mean weekly consumption (pints) } \\
\text { range }\end{array}$ & $\begin{array}{l}8.5 \pm 9.5^{\star} \\
0-50\end{array}$ & $\begin{array}{l}7.4 \pm 11.4^{\star} \\
0-64\end{array}$ & \\
\hline \multicolumn{4}{|c|}{ Physical exertion (occupational and/or leisure) } \\
\hline heavy & IO & IO & NS \\
\hline very light (sedentary) & 26 & 23 & NS \\
\hline Employed & 56 & 74 & \multirow{3}{*}{$P<0.01$} \\
\hline Unemployed & 3 & 4 & \\
\hline Retired & 28 & 20 & \\
\hline 'Chronic sick' & 13 & 2 & \multirow{4}{*}{$\begin{array}{l}P<0.01 \\
\text { NS } \\
\text { NS }\end{array}$} \\
\hline Chronic bronchitis (cigarette smokers) & $56(5 I)$ & 21 (19) & \\
\hline Hypertension & 12 & 15 & \\
\hline Diabetes mellitus & 6 & 5 & \\
\hline \multicolumn{4}{|l|}{ Family history } \\
\hline myocardial infarction & 48 & 44 & NS \\
\hline angina & 50 & 44 & NS \\
\hline sudden death & 40 & 30 & NS \\
\hline \multicolumn{4}{|l|}{ History of atheromatous disease } \\
\hline cerebrovascular accident & 8 & 6 & \multirow{4}{*}{$\begin{array}{l}\text { NS } \\
\text { NS } \\
P<0.05 \\
\text { NS }\end{array}$} \\
\hline claudication & 10 & 14 & \\
\hline myocardial infarction & 27 & 16 & \\
\hline $\begin{array}{l}\text { angina } \\
\text { stable }\end{array}$ & 30 & 34 & \\
\hline $\begin{array}{l}\text { mean duration (weeks) } \\
\text { range (weeks) }\end{array}$ & $\begin{array}{l}237 \pm 173^{\star} \\
25-750\end{array}$ & $\begin{array}{l}180 \pm 187^{\star} \\
8-800\end{array}$ & $\begin{array}{l}\text { NS } \\
\text { NS }\end{array}$ \\
\hline unstable & 38 & 59 & $\mathrm{P}<0.01$ \\
\hline mean duration (weeks) & 3 & 4 & NS \\
\hline range (days) & $I-28$ & $I-40$ & NS \\
\hline
\end{tabular}

$\star$ Mean \pm standard deviation.

of the non-fatal myocardial infarcts (control group) alongside. The results of the inquiry into the circumstances attending sudden death are set out in Fig. I-5 and for comparison the results of the same inquiries of the non-fatal myocardial infarcts are set out alongside. But the within-group comparison of the sudden deaths are more important.
Circumstances attending death or onset of infarction

Season of year. During the period of the study (September I971 to June 1972) the numbers in the sudden death group did not vary significantly from month to month (Fig. I).

Day of week. The commonest day for sudden 


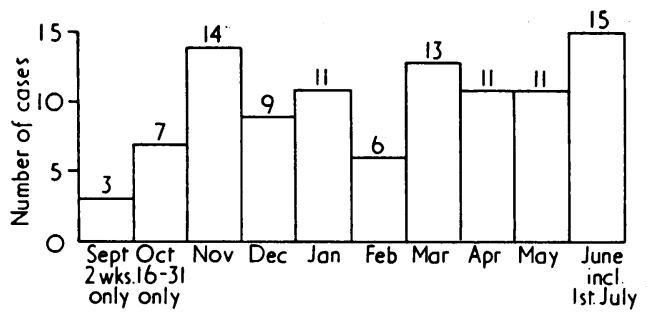

FIG. I Month of death (sudden death group only).

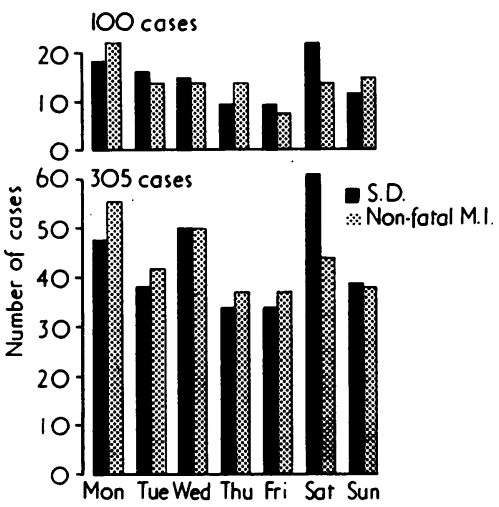

FIG. 2 Day of week in which sudden deaths and non-fatal myocardial infarctions occurred. Additional cases, not in series fully studied, added in lower part of Fig. (see text).

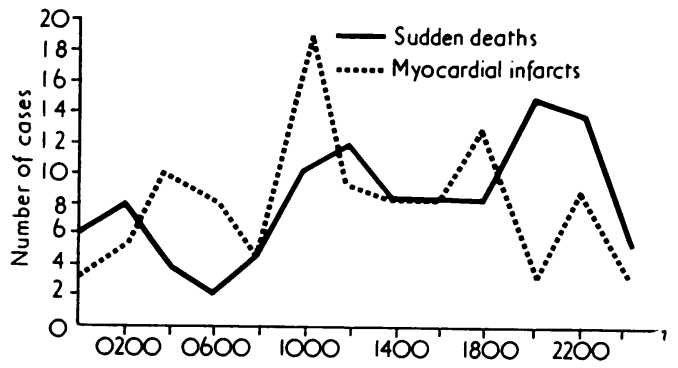

FIG. 3 Time of day of sudden deaths and non-fatal myocardial infarcts.

death was Saturday (Fig. 2) but the preponderance was not greater than could be accounted for by chance. Accordingly a further 205 coroner's necropsy records of sudden death from coronary disease were examined retrospectively and the day of the week for the death determined. From this total of 205 cases Saturday is shown to be significantly $(P<0.05)$ the most common day. This did not apply to the non-fatal myocardial infarction group.

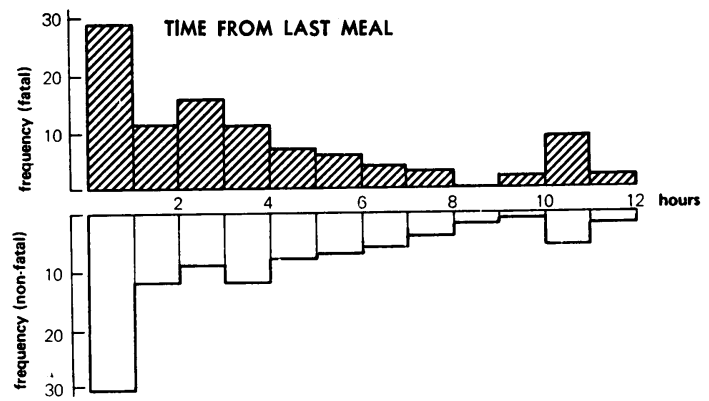

FIG. 4 Time from last meal of sudden deaths and non-fatal myocardial infarcts.

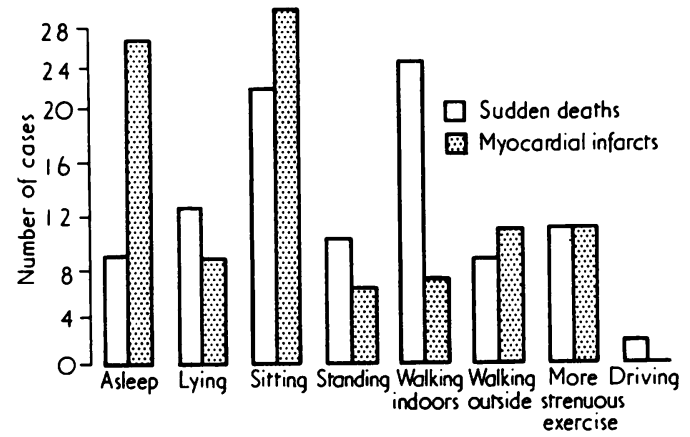

FIG. 5 Maximal activity during 5 minutes preceding onset.

Time of day. Significantly $(\mathrm{P}<0.05)$ fewer sudden deaths occurred during the night, especially between 2 a.m. and 6 a.m., than would be expected by chance. In the non-fatal myocardial infarction group no significant differences were found. The peak incidence for sudden death was in the late evening, 6-ro p.m., and for non-fatal myocardial infarction 4-6 p.m. and 8-10 a.m. (Fig. 3).

Timing and nature of preceding food and drink. Twenty-nine of the deaths and 30 of the infarcts occurred within one hour of ending a meal (Fig. 4). The number of meals consumed varied between three and four a day, so that the hour after them represents at most one-sixth of the 24 hours or, if eight hours of sleep are excluded, one-quarter of the individual's waking life. It seems therefore that it is by a little more than mere chance that nearly one-third of both sudden deaths and non-fatal infarcts occurred in that period. There was no difference in the habitual alcohol consumption and pattern of such consumption between the two groups. A larger number (18) of the sudden death group had taken alcohol in the three hours before death than of the non-fatal myocardial infarction group (9). This difference is statistically significant $(P<0.05)$ even though the numbers were rather 
small. The amount of fat and salt in the meal did not prove to have any special importance. Those who had had a high fluid intake did not have a higher incidence of pulmonary oedema.

Environmental temperature. The number of deaths and of non-fatal infarcts in the various environmental circumstances in which they took place are shown in Table 2. Few in either group occurred in either extreme of temperature, and most of both occurred in pleasantly warm surroundings. There were more sudden deaths (12) in cold circumstances, either outside or indoors, than there were cases of non-fatal myocardial infarct (7), but the difference is not significant. Of the sudden deaths in the half hour before onset, 32 had changed from warm to cold environment or vice versa ; for the nonfatal myocardial infarction group the figure was 22 . This difference did not attain statistical significance. Considerably more in both groups had changed from cold to warm surroundings than vice versa.

Physical activity preceding sudden death and nonfatal infarction. Only a minority (10) of the sudden death group were doing anything more strenuous than walking in the five minutes preceding death (Fig. 5); one was engaged in heavy exertion (carrying a coffin), not unusual for him, in these five minutes and six had performed heavy exercise in the 24 hours preceding death. Similarly only ro of the non-fatal myocardial infarction cases had been undertaking any more strenuous exertion than walking in the five minutes preceding its onset. The incidence of varying degrees of exercise did not differ between the two groups whether periods

TABLE 2 Environmental temperature at onset of sudden death and non-fatal myocardial infarction

\begin{tabular}{lcc}
\hline & Sudden deaths & $\begin{array}{l}\text { Non-fatal } \\
\text { myocardial } \\
\text { infarction }\end{array}$ \\
\hline Outdoors & & \\
$\quad$ very cold & 3 & 3 \\
cold & 3 & 4 \\
medium temperature & 4 & 5 \\
warm & 1 & 5 \\
very warm & 0 & 1 \\
$\quad$ coors & 6 & 0 \\
medium temperature & 21 & 10 \\
warm & 62 & 69 \\
very warm & 0 & 3 \\
\hline
\end{tabular}

Differences are not significant. of one, three, six, or 24 hours before death or infarction respectively were examined.

Cigarette smoking. There was no greater prevalence of a cigarette having been smoked immediately before death or infarction than would be accountable by pure chance. It is noteworthy that 85 and 82 of the sudden death and non-fatal myocardial infarction groups respectively were habitual smokers.

Psychological stress. Details of the incidence and, in the case of acute stress, of the likelihood of occurrence by chance are shown in Table 3. Twentythree of the sudden death group were reported to have experienced a significant degree of psychological stress in the 30 minutes before death as compared with eight of the non-fatal myocardial infarct group in the 30 minutes before the onset of their infarct $(P<0.02)$. Forty of the fatal and 24 of the infarct group had experienced apparently significant stress in the 24 hours (including the first 30 minutes) before death or infarction, but the difference between these figures is not statistically significant. Examples of significant stress in the sudden death cases were first attendance at surgical outpatients ( 2 cases), cystoscopy shortly beforehand, an attack by dogs ( 2 patients), a row over a game of dominoes (I patient), and a similar row over a darts match (I patient). A bus driver died after being involved in a road traffic accident, two patients died shortly after notification that their divorce had been made absolute, one died just before interview for a job when he had been out of work for some months, and one died after he had driven in a motor race for the first time in his life. No patient was recorded as having died soon after or during coitus. Twelve of the sudden deaths group had been subject to major long-term (defined as persisting for 6 months or more) psychological stress and 16 to minor, making a total of 28 . Five of these, however, also suffered acute stress in the 24 hours preceding their death, so that the significance of the long-term stress is difficult to evaluate. In the non-fatal myocardial infarction group the figures for chronic psychological stress were 15 for major and 23 for minor, totalling 38. The 'rarity' of such chronic stress was impossible to evaluate.

\section{Instantaneous deaths}

These numbered 34. They have been compared with the other 66 both in respect of their primary status and also of the various circumstances attending their deaths as previously described for the whole group. Partly because the numbers involved are small, none of the differences attained statistical significance, but these differences were mostly very small indeed. 
TABLE 3 Psychological stress in relation to sudden death and onset of non-fatal myocardial infarction

\begin{tabular}{|c|c|c|c|c|c|c|}
\hline \multicolumn{7}{|l|}{ Acute stress } \\
\hline \multicolumn{7}{|c|}{ In preceding 30 minutes } \\
\hline & $\begin{array}{l}\text { Sudden } \\
\text { death }\end{array}$ & $\begin{array}{l}\text { Rarity } \\
\text { No. }\end{array}$ & Degree & $\begin{array}{l}\text { Non-fatal } \\
\text { myocardial } \\
\text { infarction }\end{array}$ & $\begin{array}{l}\text { Rarity } \\
\text { No. }\end{array}$ & Degree \\
\hline None/minimal & 77 & & & 92 & & \\
\hline Moderate & 18 & $\begin{array}{r}10 \\
6 \\
2\end{array}$ & $\begin{array}{l}++ \\
+ \\
+\end{array}$ & 5 & $\begin{array}{l}3 \\
2\end{array}$ & $\begin{array}{l}++ \\
+\end{array}$ \\
\hline Severe & 5 & 5 & $+t+$ & 3 & 3 & +++ \\
\hline \multicolumn{7}{|c|}{$x^{2}=13.8$ with 6 degrees of freedom. $\mathrm{P}<0.02$} \\
\hline \multicolumn{7}{|c|}{ In preceding 24 hours } \\
\hline & $\begin{array}{l}\text { Sudden } \\
\text { death }\end{array}$ & $\begin{array}{l}\text { Rarity } \\
\text { No. }\end{array}$ & Degree & $\begin{array}{l}\text { Non-fatal } \\
\text { myocardial } \\
\text { infarction }\end{array}$ & $\begin{array}{l}\text { Rarity } \\
\text { No. }\end{array}$ & Degree \\
\hline None/minimal & 60 & & & 76 & & \\
\hline Moderate & 30 & $\begin{array}{l}17 \\
10\end{array}$ & $\begin{array}{l}++ \\
+\end{array}$ & 18 & $\begin{array}{r}12 \\
6\end{array}$ & $\begin{array}{l}++ \\
+\end{array}$ \\
\hline Severe & 10 & $\begin{array}{l}3 \\
7 \\
3\end{array}$ & $\begin{array}{l} \pm \\
++ \\
++\end{array}$ & 6 & $\begin{array}{l}3 \\
\mathbf{I} \\
2\end{array}$ & $\begin{array}{l}+++ \\
++ \\
+\end{array}$ \\
\hline
\end{tabular}

Chronic stress

\begin{tabular}{lll}
\hline & $S D$ & Non-FMI \\
None'minimal & 72 & 62 \\
Moderate & 16 & 23 \\
Severe & 12 & 15 \\
$\chi^{2}=2.87$ with 6 degrees of freedom. $\mathrm{P}=0.9$ & \\
\hline
\end{tabular}

$+++=$ likelihood of occurrence less than once in a month.

$++=$ likelihood of occurrence at least once in a month but not weekly.

$+=$ likelihood of occurrence at least weekly but not daily.

$\pm=$ likelihood of occurrence at least once daily.

Only the higher proportions of the instantaneous *. cases who had sustained previous infarction (16/34: II/66), who were non-smokers $(7 / 34: 8 / 66)$ or nearly or wholly teetotal $(20 / 34: 16 / 66)$ are worthy of comment.

\section{Post mortem studies}

Heart weight was on average abnormally great (462 grams).

Preterminal abnormalities. Atheroma was present in at least two arteries of all the roo hearts examined. In 98 of them at least one of the three main vessels had 75 per cent or more of the lumen occluded by atheroma or thrombosis. Old thrombosis was present in $31: 21$ in the right coronary artery, 10 in the left anterior descending, and six in the left circumflex. In six cases more than one thrombus was present. Old infarction was present in 45 cases and old ischaemic fibrosis in a further 19. Severe atheroma (at least $75 \%$ narrowing) was present in
98 cases, affecting the right coronary artery in 54, the left anterior descending in 72 , and the left circumflex in $4 \mathrm{I}$.

Terminal abnormalities. Recent thrombosis was found in 38, affecting the right coronary artery in 19, the left anterior descending in 12, and the left circumflex in 9. In two cases two vessels were thrombosed: 17 of the 38 also had recent infarcts. Recent infarction was recognizable in 27 , predominantly anterior in $\mathrm{Ir}$, predominantly posterior in 14, and predominantly lateral in two. In ro of the 27 no new thrombus was found. In nine there were both old and new infarcts. In 52 cases, therefore, neither new thrombus nor new infarction was found: 24 of these showed severe atheroma only and $28 \mathrm{had}$ old thrombus or infarcts as well.

Relationship between post-mortem findings and circumstances attending death

In an effort to relate post-mortem findings to the 
TABLE 4 Acute and chronic pathological changes in instantaneous and sudden deaths

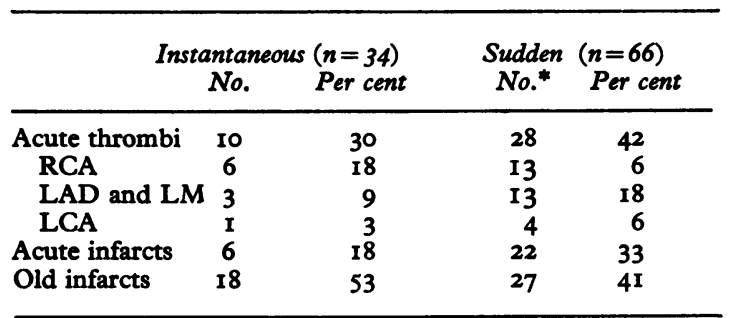

LM=left main.

* In 2 cases 2 vessels had acute thrombi.

circumstances occurring before death the presence or absence of new thrombi and the presence or absence of new infarction were correlated in $\chi^{2}$ tables with the percentage over or under weight, the presence or absence of psychological stress before death, the timing of attack from last meal, the last consumption of alcohol or smoking of last cigarette, and with the smoking history both recent and maximal in the past. There was no correlation between any of these circumstances and the post-mortem findings.

There was, however, a relationship between the degree of narrowing in the circumflex and right coronary arteries and the duration of symptoms preceding death. It was an inverse one, those with severe stenosis or total occlusion, whether recent or old, of these vessels tending to die instantaneously. This was not seen in those in whom the anterior descending artery was severely affected by atheroma. Further details are given in Table 4. Those patients who had severe atheroma in the right coronary artery, on the other hand, had on average a longer history of stable angina and an angina of greater severity than did those with severe occlusion of either branch of the left.

\section{Discussion \\ Material and methods}

The selection of coroner's cases for the sudden death series was decided upon partly as a relatively easy way of gaining access to a useful number of cases in whom the interval between onset of symptoms and death was as short as possible, partly to ensure that necropsy findings were always available and that these would be performed consistently by one or other of only two pathologists. Mostly these objects were attained. In 27 of the cases the interval between onset to death exceeded one hour, so that they were not all very sudden, but the range has proved useful for analysis and especially for comparison of the post-mortem findings.
For many of the circumstances studied the cases have acted as their own controls, but the 100 nonfatal infarcts have served a purpose as an accessory control group. The basic differences between them were unexpected. Thus the smaller numbers in the coroner's cases from social classes I and II - with the higher incidence of bronchitis and of unemployment which may be related thereto - were a surprise, especially as the distribution for social classes in the infarct group was the same as in the city as a whole. One explanation may be that the sudden death cases resided in areas spread all over the city whereas the infarct group were in greater part $(67 \%)$ from the eastern half, in which the Royal Victoria Infirmary is situated. But both halves of the city have quite mixed populations. Another explanation may be that doctors more readily refer cases of sudden death from social classes IV and V to the coroner. Nevertheless, it is noteworthy that, apart from weight $(7 \%$ excess in the non-fatal myocardial infarct group only), the recognized coronary risk factors - family history, hypertension, habits of smoking and of exercise, and incidence of diabetes were closely similar in the groups. The sudden death group had had more previous infarcts and the non-fatal myocardial infarct group had suffered more unstable angina. Perhaps these facts should occasion no surprise. It is interesting that the subjects in the sudden death group were not overweight, unlike the young soldiers in French and Dock's series (1944), though Moritz and Zamchek (1946), who studied 200 young soldiers who died suddenly and did not find them to be overweight, have cast a doubt on the validity of the figures for weight in French and Dock's series.

Because of the basic differences between the two groups the infarct group was divided into those who if they had died would have had a post-mortem at the Newcastle General Hospital or at the Royal Victoria Infirmary, and these groups were compared with General Hospital and Victoria Infirmary necropsies in $\chi^{2}$ tables for all the factors examined. The bias in this control group did not appear to affect the results significantly. A number of cross references were also made to determine if the predominance of the lower social classes in the fatal group may have influenced results in other ways: but this was found not to be so.

\section{Season and environmental temperature}

The investigation did nothing to confirm the popular impression that sudden death from ischaemic heart disease occurs more often in winter and on sudden changes of environmental temperature, especially to cold. This latter finding is perhaps 
surprising, considering how often anginal pain is precipitated by such exposure.

\section{Day of week, time of day, timing and nature of preceding food and drink}

The association of sudden death with the evening (6-10 p.m.), with the hour after a meal, and with a large consumption of alcohol is interesting and suggests that these may form a cluster of circumstances of special danger. The association, significant at the five per cent level, also with Saturday (when in N.E. England social drinking is at its maximum) lends support to this hypothesis. Whether these factors operate through an increased cardiac output, an increased blood volume, increased catecholamines in the blood or a hypercoagulable state of it, or a combination of these and other factors is an open question. The current investigation can provide no evidence towards the answer. It is not, however, as might have been supposed, through the development of severe left ventricular failure with pulmonary oedema (for it was large quantities of beer which were consumed), for this complication was not found with any special frequency in the cases in question.

\section{Smoking}

The evidence that cigarette smoking is a highly important factor leading to coronary artery disease and the risk of death from it, including sudden death, is very strong (Doyle et al., 1964; Dorn, 1959; British Medical fournal, 197I). The prevalence of the habit was considerable, over 80 per cent, but there was no significant difference between the sudden death and the non-fatal myocardial infarct groups. It might be thought that the actual smoking of a cigarette would frequently provoke sudden death by setting off dangerous cardiac arrhythmias, especially as Bellet et al. (1970) have shown in dogs that inhalation of cigarette smoke lowers the threshold for ventricular fibrillation. The present investigation does not support this concept. Equally it does not in any way absolve cigarettes from their role as a serious risk factor.

\section{Activity, sleep, and psychological stress}

In only a small proportion was exercise of unusual severity undertaken in the period before death or myocardial infarction. The sudden death group had been a little more active than the infarct group but no more than to the extent of sitting or moving slowly about the house. Conflicting views on exercise have been expressed. Boas (1939), French and Dock (1944), Yater et al. (1948), and Hansen (I968) claimed that exercise of unusual severity was commoner before sudden death from coronary artery disease. Phipps (1936), Kuller, Lilienfeld, and Fisher (1967), and Wikland (1971) were of the opposite opinion. The most recent report by Friedman et al. (1973) also stressed the large proportion (37\%) who were engaged in strenuous activity immediately or very soon before their sudden death but in their case too the exercise was also most often not unusual for the individuals concerned. The matter has considerable medico-legal importance but in relation to the total numbers involved is of little account.

In the current series the number dying during sleep, only nine, was remarkably small, about the same proportion as in the series of Friedman et al. (1973). Several had been awakened by chest pain or dyspnoea before demise, but their number was not enough to distort the general pattern of relative safety between midnight and 6 a.m. (Fig. 3). Lown et al. (1973) showed in 35 out of 45 patients that ventricular ectopic beats during waking hours diminished often to half the number during sleep. Perhaps because of this the danger in sleep of 'unheralded arrhythmic' sudden death appears to be quite small, much smaller indeed than the risk of classical infarction. Diminished psychological stress and consequent diminished release of catecholamines presumably play a part in this.

Psychological stress has in this investigation turned out to be an important factor. There is no doubt, of course, that patients and their relatives are suggestible and keen to find stress factors to account for disasters as dramatic as sudden death and myocardial infarction. Nevertheless, the acute stresses documented were described to A.M. in fresh and circumstantial detail, after which their unusualness and in some cases uniqueness were established by further careful inquiry (Table 3 ). In the sudden death cases this stress factor has operated in a graded and interesting way. Subacute psychological stress - that is, in the 24 hours preceding onset - was more frequent (40:24) among the sudden death than the non-fatal myocardial infarction group, but not to a significant degree. Really acute psychological stress - that is, in the 30 minutes preceding onset - was significantly more frequent $(23: 8) \quad(P<0.02)$. The significance of physical activity and acute psychological stress are therefore compatible with the thesis that the release of catecholamines is the determining factor, as has been suggested by the studies of Taggart, Parkinson, and Carruthers (1972). The type of emotion involved in the present series was too diverse for one to be able to conclude with any certainty that it was the release of adrenaline rather than noradrenaline or vice versa which carried the greater risk. It may well 
be that most often both are involved. The results confirm statistically the anecdotal information collected by Engel (197I).

The importance of chronic psychological distress, on the other hand, cannot be evaluated here. Its prevalence the groups and we made no attempt to compare these two groups of ischaemic heart disease cases with a matched group of 'normal' subjects free from symptoms of ischaemic heart disease as was done by Friedman et al. (1973).

\section{Post-mortem studies}

The post mortem findings are similar to those reported by French and Dock (1944), Weinberg and Halpern (1959), Crawford et al. (196r), Kuller et al. (1967), Hansen (1968), and Spain and Bradess (1970). In all these series diffuse atheroma was reported in two or all of the main coronary vessels; in nearly all of those who died suddenly from coronary artery disease, at least one of the vessels had its lumen narrowed by 75 per cent or more. The incidence of recent infarcts in previously reported series was usually low - French and Dock 19 per cent, Kuller et al. 12 per cent, and Hansen ro per cent; there was a higher incidence of recent thrombi - French and Dock 36 per cent, Crawford et al. 55 per cent, Kuller et al. 19 per cent, Hansen 69 per cent, and Spain and Bradess 19 per cent. In our series of 100 sudden deaths infarcts were found in 27 and thrombi in 38 . The relatively higher incidence of recent infarction in our series compared with the others is probably due to the macroscopic staining method used which delineates infarcts earlier than can be done by simple inspection.

\section{Sites of infarction}

The distribution of recognizable new infarcts (27 cases) was anterior in eight cases $(28.6 \%)$, posterior in $14(51.9 \%)$, septal in three (11.1\%), and lateral in two $(7.4 \%)$. This may be compared with the two carefully studied series of 357 cases quoted by PIotz (1957) of fatal infarcts (not necessarily sudden) where the distribution was anterior in 190 $(53 \%)$, posterior in $109(31 \%)$, septal in five ( $1.4 \%)$, mixed in 45 (12.6\%), and right ventricular in eight $(2 \%)$. Thus in sudden death cases posterior infarcts are unusually common.

\section{Sites of thrombi}

Closely concordant with this is the distribution of thrombi in the different coronary arteries. Plotz (1957) aggregated 1495 fatal cases of coronary occlusion, again not predominantly sudden, and the vessels involved were left anterior descending $834(56 \%)$, with $71(5 \%)$ in the left main artery, $379(25 \%)$ in the right coronary, and 2 II (14\%) in the left circumflex. In Crawford et al.'s (I96I) series, not included in the above and consisting of sudden death cases only, the proportions were left anterior descending $27(57 \%)$, right $20(31 \%)$, and circumflex $8(12 \%)$. In the present series the proportions were left anterior descending $12(30 \%)$, right 19 $(47.5 \%)$, and circumflex $9(22.5 \%)$. Thus in this series the sudden death was more often associated with recent occlusion of the right coronary artery and with posterior infarction than of the left anterior descending and associated anterior infarction. Presumably this reflects the fact that interference with the pacemaking mechanism and with the conducting system is a particularly dangerous complication of coronary atheromatous disease, as has been stressed by James (1972).

\section{Amount of warning}

Fifty of the patients had had their coronary artery disease diagnosed during life and a further 16 had had symptoms compatible with coronary artery disease but undiagnosed. In only 34, therefore, was death totally unexpected. In contradistinction to Kuller et al.'s (1967) experience in Baltimore, where as many as 23 per cent had seen a physician within one week of their death, in the present series only three had done so.

The very high mortality in the first hour, already well documented by McNeilly and Pemberton (1968) and Fulton, Julian, and Oliver (1969), is also well brought out in the present series where, during a period of nine months in a city of 300,000 inhabitants, some 73 males died within an hour of the onset of symptoms. The place of mobile coronary care units in preventing these deaths is clearly limited. The fact that half of the cases had never seen a doctor about their malady emphasizes the great importance of primary prevention. For the other half, modern methods of secondary preventive treatment may offer some hope. Recent developments in coronary arterial surgery possibly will fortify that hope.

Finally, having separated the many circumstances studied into those which did and those which did not contribute to sudden death, we are tempted to try to explain the differences in terms of possible mechanisms. If the time of day and the striking importance of acute psychological stress, especially as it relates to those deaths which were instantaneous (Table 4), are taken into account the factor of catecholamine levels in the blood obvi- 
ously stands out, even though these levels could not be measured.

In favour of increased cardiac work leading to myocardial anoxia as a factor are the associations with activity and with the hour after a meal, especially if alcohol was consumed, and with acute psychological stress. Against its importance is the infrequency of really strenuous activity and the negative correlation with exposure to cold, which may be presumed to raise the blood pressure, and the absence of any correlation with the actual smoking of a cigarette.

The tendency for sudden death to occur in the first hour after a meal, especially an evening meal, might, however, suggest that it was the fat content which was important. It might well be so, though, on inquiry, the meals did not have an exceptionally high fat content. If it is so, how does the fat content operate ? If it were simply a question of a high level of free fatty acids in the blood stream the early morning fasting state should have also been lethal, but it was not. If free fatty acids are important factors they would seem also to need to be associated with a high level of catecholamines or some extra physical stress on the myocardium, or both. Neutral fat, split or unsplit, and levels of glucose and insulin could all have a bearing on states of 'hypercoagulability' and states of fibrinolysis as well as on myocardial metabolism and excitability, and there seems little point in speculating further about them.

We thank Dr. D. R. Appleton for preparing the computerized analysis; Drs. J. A. J. Ferris, Royal Victoria Infirmary, and B. J. Smith, Newcastle General Hospital, for their post-mortem examinations; H. M. Coroner and his principal officer, City of Newcastle upon Tyne, for permission and help in identifying cases; Drs. S. E. Beckett and C. E. Johnson for obtaining additional information from coroner's and hospital records; and the relatives and patients who have been so helpful in providing the detailed information on which the study is based. The investigation was supported by the Research Fund of the United Newcastle upon Tyne Hospitals.

\section{References}

Baroldi, G. (1965). Acute coronary occlusion as a cause of myocardial infarct and sudden coronary heart death. American fournal of Cardiology, 16, 859.

Bellet, S., Fleischman, D., Roman, L., and DeGuzman, N. (I970). The effect of cigarette smoke inhalation on the ventricular fibrillation threshold. Circulation, 42, Suppl. III, 135 .

Boas, E. P. (1939). Angina pectoris and cardiac infarction from trauma or unusual effort with a consideration of certain medicolegal aspects. Fournal of the American Medical Association, $112,1887$.
British Medical fournal (1971). Trial of clofibrate in the treatment of ischaemic heart disease: 5 year study by physicians of the Newcastle upon Tyne region. 4, 767.

Camps, F. E. (1969). Recent Advances in Forensic Pathology. Churchill, London.

Crawford, T., Dexter, D., and Teare, R. D. (196I). Coronaryartery pathology in sudden death from myocardial ischaemia. Lancet, $1,181$.

Dorn, H. F. (1959). Tobacco consumption and mortality from cancer and other diseases. Public Health Reports, 74, 581.

Doyle, J. T., Dawber, T. R., Kannel, W. B., Kinch, S. H., and Kahn, H. A. (1964). The relationship of cigarette smoking to coronary heart disease; the second report of the combined experiences of the Albany, New York, and the Framingham, Massachusetts, studies. Fournal of the American Medical Association, 190, 886.

Engel, G. L. (1971). Sudden and rapid death during psychological stress. Annals of Internal Medicine, 74, $77 \mathrm{I}$.

Friedman, M., Manwaring, J. H., Rosenman, R. H., Donlon, G., Ortega, P., and Grube, S. M. (1973). Instantaneous and sudden deaths: clinical and pathological differentiation in coronary artery disease. Fournal of the American Medical Association, 225, 1319.

French, A. J., and Dock, W. (1944). Fatal coronary arteriosclerosis in young soldiers. Fournal of the American Medical Association, 124, 1233.

Fulton, M., Julian, D. G., and Oliver, M. F. (1969). Sudden death in myocardial infarction. Circulation, 39-40, Suppl. IV, 182.

Haeren, J. W. (197I). Platelet aggregates in intramyocardial vessels of patients dying suddenly and unexpectedly of coronary artery disease. In International Society on Thrombosis and Haemostasis, II Congress, Oslo I97I, p. 128.

Hansen, J. P. H. (1968). Coronary death in younger persons. Danish Medical Bulletin, 15, $30 \mathrm{r}$.

James, T. N. (1972). Sudden death related to myocardial infarction. Circulation, 45, 205.

Kuller, L., Lilienfeld, A., and Fisher, R. (1967). An epidemiological study of sudden and unexpected deaths in adults. Medicine, 46, 34r.

Lown, B., Tykocinski, M., Garfein, A., and Brooks, P. (1973). Sleep and ventricular premature beats. Circulation, 48, 691.

McNeilly, R. H., and Pemberton, J. (1968). Duration of last attack in 998 fatal cases of coronary artery disease and its relation to possible cardiac resuscitation. British Medical Fournal, 3, 139.

Mitchell, J. R. A., and Sharp, A. A. (1964). Platelet clumping in vitro. British fournal of Haematology, 10, 78.

Moritz, A. R., and Zamchek, N. (1946). Sudden and unexpected death in young soldiers. Archives of Pathology, 42, 459.

Phipps, C. (1936). Contributory causes of coronary thrombosis. Fournal of the American Medical Association, 106, 76 r.

Plotz, M. (1957). Coronary Heart Disease, p. 88. Cassell, London.

Spain, D. M., and Bradess, V. A. (1970). Sudden death from coronary heart disease: survival time, frequency of thrombi, and cirarette smoking. Chest, 58, 107.

Taggart, P., Parkinson, P., and Carruthers, M. (1972). Cardiac responses to thermal, physical and emotional stress. British Medical fournal, 3, 7r. 
Weinberg, S. B., and Halpern, M. (1959). Circumstances related to sudden and unexpected death in coronary heart disease. In Work and Heart, p. 288. Ed. by F. F. Rosenbaum and E. L. Belknap. Hoeber, New York.

Wikland, B. (I97I). Medically unattended fatal cases of ischaemic heart disease in a defined population. Acta Medica Scandinavica, Suppl. 524.

Yater, W. M., Traum, A. H., Brown, W. G., Fitzgerald,
R. P., Geisler, M. A., and Wilcox, B. B. (1948). Coronary artery disease in men 18-39 years of age. Report of 866 cases, 450 with necropsy examinations. American Heart fournal, 36, 334, 381, and 683 .

Requests for reprints to Dr. H. A. Dewar, 27 Montagu Avenue, Gosforth, Newcastle upon Tyne NE3 $4 \mathrm{HY}$. 